\title{
Invasive fungal infection in children with hematologic malignancy
}

\author{
Belgin Gülhan ${ }^{1}$, Saliha Kanık-Yüksek¹, Aslınur Özkaya-Parlakay ${ }^{1}$, Neşe Yaralı², \\ Hasan Tezer ${ }^{1}$, Namık Yaşar Özbek ${ }^{2}$ \\ Departments of ${ }^{1}$ Pediatric Infectious Diseases and ${ }^{2}$ Pediatric Hematology and Oncology, Ankara Child Health and Diseases \\ Hematology Oncology Training and Research Hospital, University of Health Sciences, Ankara, Turkey. \\ E-mail: docbelgin@yahoo.com \\ Received: 8th March 2019, Revised: 15th May 2019, Accepted: 16th May 2019
}

SUMMARY: Gülhan B, Kanık-Yüksek S, Özkaya-Parlakay A, Yaralı N, Tezer H, Özbek NY. Invasive fungal infection in children with hematologic malignancy. Turk J Pediatr 2019; 61: 159-165.

Despite improvements in diagnosis and treatment, invasive fungal infection (IFI) is still a major cause of morbidity and mortality in immunocompromised patients. In patients with hematologic malignancy, the most invasive fungal infections are caused by Candida and Aspergillus fumigatus. This study was designed retrospectively to summarize data in pediatric patients with acute lymphoblastic leukemia (ALL) and acute myeloblastic leukemia (AML) including risk factors for IFI, epidemiological and clinical features and treatment choices involving combination therapy from January 2006 through December 2014. We analyzed the records of 154 pediatric patients (125 ALL and 29 AML) receiving chemotherapy for hematologic malignancy. During follow-up 60 IFI episodes were observed. IFI episodes were observed more common in AML, compared to ALL $(\mathrm{p}=0.002)$. Among 60 IFI episodes, eight were proven, seven were probable and 45 were possible IFI episodes. Galactomannan antigen was investigated in 37 IFI episodes and found positive in seven probable IFI episodes. Fungemia was detected in seven patients with proven IFI and the most common microorganism was non-albicans candida spp. The most common antifungal drug was fluconazole $(14.8 \%)$. A total of 29 patients $(48 \%)$ had received empirically liposomal amphotericin $B$ and 10 patients $(16.6 \%)$ had received caspofungin. Crude mortality was $10.3 \%$ and attributable mortality was $6.4 \%$ during the study period. Invasive fungal infections continue to be a major cause of morbidity and mortality in children with hematologic cancer. The most common isolated agent from hemoculture was non-albicans Candida spp.

Key words: fungal infection, leukemia, children.

Despite improvements in diagnosis and treatment, invasive fungal infections (IFI) are still a major cause of morbidity and mortality in immunocompromised patients. ${ }^{1,2}$ In children, the true incidence and outcome of IFI are more difficult to determine because of the growing trend toward more invasive chemotherapeutic regimens, the development and introduction of new antifungal drugs and prophylactic antifungal using, and differences in design and patient populations among various studies. ${ }^{3}$ In patients with hematologic malignancy, most invasive fungal infections (IFI) are caused by Candida and Aspergillus fumigatus. ${ }^{4-6}$
Factors that appear to be associated with IFIs in both children and adults in this severely immunosuppressed population are the underlying malignancy (mainly acute leukemia), presence of profound and longlasting neutropenia, high intensity of the chemotherapeutic regimen, hematopoietic stem cell trasplantation (HSCT), and previous antibiotic therapy. ${ }^{4-6}$

In pediatric patients caspofungin and liposomal amphotericin B (L-AmB) are the first-line empirical antifungal agents. Therapy with coadministration of two or three antifungals 
has not been approved by clinicians, and there is no support from randomized-controlled clinical trials. ${ }^{7}$

This study was designed retrospectively to summarize data in pediatric patients with acute lymphoblastic leukaemia (ALL) and acute myeloblastic leukemia (AML) including risk factors for IFI, epidemiological and clinical features and treatment options involving combination therapy.

\section{Material and Methods}

\section{Data collection}

Clinical parameters of all episodes of febrile neutropenia (FN) in children and adolescents admitted to the University of Health Sciences, Ankara Child Health and Diseases Hematology Oncology Training and Research Hospital from January 2006 through December 2014 were analyzed.

A data sheet for evaluating each episode was filled out including: 1) patient's age, sex, type of leukemia, and stage (remission or relapse/progressive disease), chemotherapy regime of the patient (induction/reinduction or maintenance), 2) presence of any intravenous device, day of catheter, 3) clinical assessment, highest axillary temperature on first day, blood pressure and signs and symptoms indicative of any clinically identifiable infectious focus (e.g., presence of hypotension, fever over $39^{\circ} \mathrm{C}$, paleness, etc.), 4) laboratory examination; hemoglobin level, platelet count, absolute neutrophil count (ANC), absolute monocyte count (AMC), quantitative serum C-reactive protein (CRP), 5) granulocyte stimulating factor treatment, 6) number of days since the end of the last chemotherapy and number of days since the first chemotherapy, 7) prophylactic antibiotic treatment, 8) catheter and peripheral blood culture results, chest X-ray and thorax tomography findings, 9) galactomannan (GM) antigen levels, 10) and clinical infection were noted.

The study was approved by the ethical board of the medical center (Protocol number: 2013/05719.11.2013).

\section{Definitions}

Febrile neutropenia is the occurrence of fever during a neutropenic episode. Neutropenia is defined as a neutrophil count (ANC) of $<500$ cells $/ \mathrm{mm}^{3}$, or an ANC expected to decrease to $<500$ cells $/ \mathrm{mm}^{3}$ during the next 48 hours. ${ }^{8,9}$ Fever is defined as a single oral temperature measurement of $>38.3^{\circ} \mathrm{C}$ $\left(101^{\circ} \mathrm{F}\right)$ or a temperature of $>38.0^{\circ} \mathrm{C}\left(100.4^{\circ} \mathrm{F}\right)$ sustained over a 1 hour period according to the Infectious Diseases Society of America (IDSA) Clinical Practice Guideline. ${ }^{8}$ Fever is defined as an oral temperature of $>38.5^{\circ} \mathrm{C}$ or two consecutive readings of $>38.0^{\circ} \mathrm{C}$ for 2 hour according to European Society of Medical Oncology (ESMO) Clinical Practice Guidelines. ${ }^{9}$

The IFI diagnosis was defined according to revised definitions of the European Organization for Research and Treatment of Cancer/Invasive Fungal Infections Cooperative Group and the National Institute of Allergy and Infectious Diseases Mycoses Study Group. Proven IFI was defined as fungal growth in normally sterile areas. The probable IFI was defined as presence of host factors, clinical features, and mycological evidence, and possible IFI only in the presence of proper host factors and sufficient clinical evidence compatible with IFI. ${ }^{10}$

Diagnosis of catheter related bloodstream infection (CRBSI) is based on the following: the presence of a central venous catheter (CVC); signs of catheter insertion site infection, clinical symptoms and signs of bacteremia; resolution of the symptoms and signs of bacteremia after removal of the suspect CVC; positive blood culture; and growth of the same organism from the catheter. 11

Febrile neutropenia was empirically treated with piperacillin-tazobactam, ceftazidim, cefaperazone-sulbactam or meropenem with/without amikacin. If the patient was hemodynamically unstable, a glycopeptide antibiotic was empirically added. Empirical treatment with liposomal amphotericin B (L-AmB, 3-5 mg/kg/day) and caspofungin (70 $\mathrm{mg} / \mathrm{m}^{2}$ for first day and $50 \mathrm{mg} / \mathrm{m}^{2}$ from second day to end of the therapy) were initiated for patients suspicious of a fungal infection or when fever was not decreased on fourth or fifth day of neutropenic fever episode. Thorax computed tomography (CT) was performed in addition to sinus $\mathrm{CT}$ if there was a clinical suspicion of fungal sinusitis. If radiologic and/or microbiologic investigations were indicative of an invasive aspergillosis infection voriconazole 
Table I. Disease Characteristics of the Patient according to Type of Invasive Fungal Infection.

\begin{tabular}{lccccc}
\hline Characteristics & $\begin{array}{c}\text { Proven } \\
(\mathrm{N}: 8)\end{array}$ & $\begin{array}{c}\text { Probable } \\
(\mathrm{N}: 7)\end{array}$ & $\begin{array}{c}\text { Possible } \\
(\mathrm{N}: 45)\end{array}$ & $\begin{array}{c}\text { Total } \\
(\mathrm{N}: 60)\end{array}$ & P value \\
\hline $\begin{array}{l}\text { Age at diagnosis, years } \\
\text { Disease status, n (\%) }\end{array}$ & $9.1 \pm 4.7$ & $3.8 \pm 4.3$ & $9.3 \pm 5.8$ & $8.7 \pm 5.7$ & 0.056 \\
$\quad$ & & & & & \\
$\quad \begin{array}{l}\text { Relapse/progressive } \\
\quad \text { Remission }\end{array}$ & $2(25.0)$ & $4(57.1)$ & $31(68.9)$ & $37(61.7)$ & 0.815 \\
$\begin{array}{l}\text { Chemotherapy, n (\%) } \\
\quad \text { Intensive }\end{array}$ & $6(75.0)$ & $3(42.9)$ & $14(31.1)$ & $23(38.3)$ & \\
$\quad$ Consolidation & $8(100.0)$ & $5(71.4)$ & $34(75.6)$ & $47(78.3)$ & 0.220 \\
$\quad$ No chemotherapy & - & $2(28.6)$ & $7(15.6)$ & $9(15.0)$ & \\
Neutropenia, n (\%) & - & - & $4(8.9)$ & $4(6.7)$ & \\
$\quad \begin{array}{l}\text { Severe }\left(0-500 / \mathrm{mm}^{3}\right) \\
\quad \text { Profound }\left(<100 / \mathrm{mm}^{3}\right)\end{array}$ & $8(100.0)$ & $5(71.4)$ & $42(93.3)$ & $55(91.7)$ & 0.067 \\
\hline
\end{tabular}

(8 $\mathrm{mg} / \mathrm{kg}$ for first day and for the maintenance $6 \mathrm{mg} / \mathrm{kg} /$ day) treatment was initiated. In patients with normal thorax CT and negative galactomannan, L-AmB or caspofungin was selected as the first-line agent. In patients with unfavourable outcome, the second antifungal agent was added. If the patient had been receiving caspofungin, voriconazole or $\mathrm{L}-\mathrm{AmB}$ was added as the second agent. In those patients who had been receiving voriconazole, L-AmB or caspofungin was added. The antifungal agent was switched according to the antifungal susceptibility results. Successful outcome was defined by the improvement of clinical signs and symptoms, findings of clinical imaging and either mycological findings or serological tests.

The chemotherapy protocols were defined as intensive or non-intensive according to the severity of expected medullary depression. Induction-consolidation therapies for acute lymphoid/myeloid leukemia (ALL/AML), relapse leukemia were considered as intensive chemotherapy. Maintenance therapies were defined as non-intensive treatments.

\section{Statistical analysis}

The statistical analysis was performed using SPSS 21.0 program package. Comparison between categorical variables was performed by two sample $t$ test or the Fisher exact test. Difference between median values of continuous variables was assessed by the Mann-Whitney U test. All tests were 2-tailed and a $P$ value of $<0.05$ was considered the threshold for statistical significance.

\section{Results}

A total of 154 pediatric patients (125 patients with ALL, 29 patients with AML) were included in the study; 352 neutropenic fever episodes were observed in 154 patients. The median age at diagnosis was 8.07 years (range 6 months-18 years). Sixty IFI episodes were observed in 51 patients during follow-up period. Among sixty IFI episodes; 8 (13.3\%) were proven, 7 (11.7\%) were probable and 45 (75\%) episodes were possible IFI. Two patients had four possible, 1 patient had 2 proven, 1 patient had 2 probable and 1 patient had 2 possible IFI episodes.

Invasive fungal infection episodes were observed more common in AML patients $(23 / 29 ; 79.3 \%)$ compared to ALL patients $(37 / 125 ; 29.6 \%$; $\mathrm{p}=0.002$ ). Neutropenia was observed in all episodes of IFI. Fifty-five patients had severe neutropenia $\left(0-500 / \mathrm{mm}^{3}\right)$ and 41 of them had profound neutropenia. Five had moderate $\left(500-1,000 / \mathrm{mm}^{3}\right)$ neutropenia. Most of the IFI episodes were observed during an induction phase of chemotherapy $(51 / 60 ; 85 \%)$. None of the patients had taken antifungal prophylaxis (Table I). Episodes with IFI had a higher CRP and lower platelet counts when compared to non-IFI patients $(\mathrm{p}=0.019$ and 0.005 , respectively; Table II). Galactomannan (GM) antigen was investigated in 37 IFI episodes and was found positive $(>0.5 \mathrm{ng} / \mathrm{ml})$ in seven probable IFI episodes and negative in 30 IFI (6 proven, 24 possible) episodes.

The most frequent site of infection was the lung in 51 IFI episodes (85\%). Seven IFI episodes 
Table II. Risk Factors for Developing Invasive Fungal Infection in Episodes.

\begin{tabular}{lccc}
\hline Laboratory values at diagnosis* & IFI episodes & Non-IFI episodes & P value \\
\hline ANC, $/ \mathrm{mm}^{3}$ & $90(0-1,500)$ & $100(0-1,500)$ & NS \\
CRP, $\mathrm{mg} / \mathrm{dl}$ & $6.41(0.18-57.01)$ & $3.21(0.01-68)$ & 0.019 \\
Platelet count, $/ \mathrm{mm}^{3}$ & $30,500(900-214,000)$ & $54,000(900-1,300,000)$ & 0.005 \\
Hemoglobin, g/dl & $8.75(5.3-12.9)$ & $9.45(5.2-14.7)$ & NS \\
\hline
\end{tabular}

*Data is presented as median (minimum-maximum)

ANC: absolute neutrophil count, CRP: C-reactive protein, IFI: invasive fungal infection

(11.7\%) had candidemia and two IFI episodes (3.4\%) had typhlitis. Twenty IFI episodes had nodules, 15 had halo sign, 6 had cavity, 2 had abscess and 9 had detected consolidation on chest CT examination.

\section{Proven and probable invasive fungal infection episodes}

Fungemia was detected in seven patients with proven IFI. The most frequent isolated fungal microorganism was non-albicans candida spp. in five $(8.3 \%)$ episodes (2 Candida krusei, 1 Candida tropicalis, 2 unknown) and in 2 (15.3\%) Candida albicans. Catheter related bloodstream infection was detected in five of seven fungemia patients and two patients with fungemia had bloodstream infection. The mean catheter day in candida species associated CRBSI positive patients was 111.4 days (range: 21-194 days).

Proven invasive aspergillosis case was a 14 year-old girl with relapse acute myeloid leukemia and chest CT of patient demonstrated consolidation on right inferior lobe with $37 \times 38 \mathrm{~mm}$ cavitary lesion. Galactomannan was negative. Combined antifungal therapy (caspofungin and voriconazole) was given for 21 days. Because bone marrow transplantation was planned for primary disease and control chest CT demonstrated abscess formation on inferior lobe of right lung, a lobectomy was performed on the side of abscess formation. Histopathological examination revealed aspergillus hyphae. However, culture did not indicate any fungal microrganism. During 3 months follow up neither aspergillus recurrence nor any surgical complication was observed after lobectomy. The proven IFI episodes are described in Table III.

Probable IFI episodes was detected in 7 episodes. All of the probable IFI cases had lower respiratory tract infection (LRTI), two patiens had cavity within the area of consolidation and one patient had cavity and fungus ball in consodilation area on chest CT examination. The other three patients had a halo sign on chest CT examination. All the probable IFI patients had positive GM antigen results in repeated times.

\section{Antifungal treatment}

We initiated antifungal agent in 149 (42.3\%) neutropenic fever episodes. The most common antifungal drug was fluconazole $(n=52 ; 14.8 \%)$. Fluconazole was mostly used for mucositis $(n=20 ; 42.5 \%)$ (e.g., oral moniliasis, aphtous stomatitis or diaper dermatitis). Secondly used antifungal in our cases was L-AmB in $45(12.8 \%)$ and caspofungin was used in 15 $(4.3 \%)$ episodes.

Twenty-nine $(48 \%)$ patients had received empirically L-AmB, 10 (16.6\%) patients had received caspofungin. Voriconazole was started in eight $(13.3 \%)$ IFI episodes according to chest CT findings consistent with Aspergillosis infection. In three (5\%) IFI episodes empirical antifungal treatment was changed, in one $\mathrm{L}-\mathrm{AmB}$ to voriconazole, in one L-AmB was changed to caspofungin and in one $\mathrm{L}-\mathrm{AmB}$ was changed to voriconazole. In nine (15\%) IFI episodes second antifungal agent was added, in six (10\%) IFI episodes voriconazole was added to L-AmB treatment, in one IFI episode voriconazole added to caspofungin, in one episode L-AmB was added to caspofungin and in one episode voriconazole was added to caspofungin treatment.

\section{Mortality}

Crude mortality was $10.4 \%(16 / 154)$ and attributable mortality was $6.5 \%(10 / 154)$ during the study period. Mortality was higher in IFI related neutropenic fever episodes $(10 / 60,16.7 \%)$, when compared to non-IFI 
Table III. Characteristics of the Patients with Proven IFI, Underlying Disease, Treatment Choices.

\begin{tabular}{|c|c|c|c|c|}
\hline Proven infection & $\begin{array}{c}\text { Underlying } \\
\text { disease }\end{array}$ & Infection site & Treatment & Prognosis \\
\hline Aspergillosis spp. & AML & Pneumonia & $\begin{array}{l}\text { L-AmB, caspofungin } \\
+ \text { vorikonazole }\end{array}$ & $\begin{array}{l}\text { Survived (exitus at } 3 \\
\text { months later, } \\
\text { Klebsiella spp. sepsis) }\end{array}$ \\
\hline Non albicans candida spp. & AML & Fungemia & Voriconazole & Survived \\
\hline Non albicans candida spp. & ALL & Fungemia & Caspofungin & Survived \\
\hline Candida albicans & ALL & Fungemia & Caspofungin & Survived \\
\hline Candida albicans & ALL & Fungemia & L-AmB & Exitus \\
\hline Candida krusei & AML & Fungemia & L-AmB & Survived \\
\hline Candida krusei & ALL & Fungemia & Fluconazole & Survived \\
\hline Candida tropicalis & ALL & Fungemia & L-AmB + caspofungin & Survived \\
\hline
\end{tabular}

ALL: acute lymphoblastic leukemia, AML: acute myeloid leukemia, IFI: invasive fungal infection, L-AmB: liposomal amphoterisin B

neutropenic fever episodes (6/292, 2.05\%; $\mathrm{p}<0.01)$. One patient with relapsed leukemia had candidemia (proven IFI) with $C$. albicans. Four patients died because of probable IFI; two of them were relapsed AML and had pneumonia with consolidation and cavity on chest CT and positive GM antigen; two patients were remission ALL, had positive GM antigen and pneumoniae. Five patients died in possible IFI group.

\section{Discussion}

The present study tried to investigate the distribution of IFI in an 8 year period in a hematology/oncology department of a major pediatric tertiary medical center. The main underlying diseases were AML (23/60, 38.3\%) and ALL $(37 / 60,61.6 \%)$. In episodes of invasive candidiasis there is an increase in nonalbicans etiologies. Of the eight proven IFIs, non-albicans Candida spp. and C. albicans were isolated in five and two episodes; respectively. Non-albicans Candida predominated in our study, which is similar to previous reports. ${ }^{3,12-14}$ Castagnola et al. ${ }^{12}$, in a 2 -year study published in 2006, prospectively evaluated 96 fungal infections defined according to the EORTC definitions. Their rate of proven infections was very high (44\%), including $27 \%$ fungemias and $17 \%$ with deep organ involvement, mainly the lung. Most of the blood infections were due to yeasts, and two were due to unidentified filamentous fungi. Their rate of probable IFIs was $18 \%$, and of possible IFIs, $38 \%$. Our rates of probable and possible IFIs were $11.6 \%$ and
$75 \%$, respectively.

Our study confirms previous observations that in children, like in adults an aggressive phase of treatment is the factor more frequently associated with the development of a fungal infection. 5,15 IFI episodes were more common in AML and induction chemotherapy, and substantially increased mortality. Other similarities with clinical features observed in adults are that non-albicans Candida are the most frequently isolated yeasts that lungs are the organs more frequently involved 15, 16,17-19. In our centre, we started to give voriconazole prophylaxis for AML patients to prevent IFI. High CRP levels and thrombocytopenia had been found mostly in IFI episodes in our study. That could be explained by high inflammation in fungal disease. The well-known risk factors for IFIs have been described as prolonged and profound neutropenia, to be in an aggressive phase of leukaemia treatment. ${ }^{12,15,20}$ Interestingly, we did not find severe neutropenia $(0-500$ cells/ $\left.\mathrm{mm}^{3}\right)$ and profound neutropenia ( $<100$ cells/ $\mathrm{mm}^{3}$ ) as a risk factor for IFI. This may be due to high number of patients with profound neutropenia. Median ANC count was similar among IFI episodes and non-IFI episodes.

In empirical treatment, caspofungin and L-AmB are the first-line agents. ${ }^{21}$ Combination antifungal chemotherapy (e.g., amphotericin B plus flucytosine and other combinations) might be considered in special situations (e.g., severe life threatening infection, compromised drug penetration in CNS infection, and complicated 
bone and joint, urinary tract, and intraabdominal infections; no grading). ${ }^{22}$

There were several limitations to our study. First, this study was designed retrospectively and represents the experience of a single centre. Consequently, our results cannot be generalized to other centres and countries. The general conditions, including building renovation, geoclimatic factors, room conditions and supportive nursing care, can be variable and therefore the risk factors cannot be standardized, particularly for invasive aspergillosis in various centres. We also lacked a control group with primary prophylaxis in our series, making it difficult to compare the incidence and mortality of IFI between groups with or without primary prophylaxis.

To conclude, IFIs continue to be a major cause of morbidity and mortality in children with hematologic malignancy. Yeast and mold infections differ significantly in incidence, organs involved, outcome, and treatment. The most isolated agent from hemoculture was nonalbicans Candida spp. and non-albicans Candida spp. are emerging as important causes of IFI in this population. This may have important implications for empirical and prophylactic treatment. Mortality due to IFIs appears to be lower than previously reported in children and adults.

\section{REFERENCES}

1. Kurosawa M, Yonezumi M, Hashino S, et al. Epidemiology and treatment outcome of invasive fungal infections in patients with hematological malignancies. Int J Hematol 2012; 96: 748-757.

2. Pagano L, Caira M, Candoni A, et al. The epidemiology of fungal infections in patients with hematologic malignancies: the SEIFEM-2004 study. Haematologica 2006; 91: 1068-1075.

3. Mor M, Gilad G, Kornreich L, Fisher S, Yaniv I, Levy I. Invasive fungal infections in pediatric oncology. Pediatr Blood Cancer 2011; 56: 1092-1097.

4. Nivoix Y, Velten M, Letscher-Bru V, et al. Factors associated with overall and attributable mortality in invasive aspergillosis. Clin Infect Dis 2008; 47: 11761184

5. Abbasi S, Shenep JL, Hughes WT, Flynn PM. Aspergillosis in children with cancer: A 34-year experience. Clin Infect Dis 1999; 29: 1210-1219.

6. Krupova Y, Sejnova D, Dzatkova J, et al. Prospective study on fungemia in children with cancer: Analysis of 35 cases and comparison with 130 fungemias in adults. Support Care Cancer 2000; 8: 427-430.
7. Lehrnbecher T, Phillips R, Alexander S, et al. Guideline for the management of fever and neutropenia in children with cancer and/or undergoing hematopoietic stem-cell transplantation. J Clin Oncol 2012; 30: 4427-4438.

8. Freifeld AG, Bow EJ, Sepkowitz KA, et al; Infectious Diseases Society of America. Clinical practice guideline for the use of antimicrobial agents in neutropenic patients with cancer: 2010 Update by the Infectious Diseases Society of America. Clin Infect Dis 2011; 52: e56-e93.

9. de Naurois J, Novitzky-Basso I, Gill MJ, Marti FM, Cullen MH, Roila F; ESMO Guidelines Working Group. Management of febrile neutropenia: ESMO Clinical Practice Guidelines. Ann Oncol 2010; 21(Suppl 5): v 252-v256

10. De Pauw B, Walsh TJ, Donnelly JP, et al; European Organization for Research and Treatment of Cancer/ Invasive Fungal Infections Cooperative Group; National Institute of Allergy and Infectious Diseases Mycoses Study Group (EORTC/MSG) Consensus Group. Revised definitions of invasive fungal disease from the European for Research and Treatment of Cancer/Invasive Fungal Infections Cooperative Group and the National Institute of Allergy and Infectious Diseases Mycoses Study Group (EORTC/MSG) Consensus Group. Clin Infect Dis 2008; 46: 1813-1821.

11. Mermel LA, Allon M, Bouza E, et al. Clinical Practice Guidelines for the Diagnosis and Management of Intravascular Catheter-Related Infection: 2009 Update by the Infectious Diseases Society of America. Clin Infec Dis 2009; 49: 1-45.

12. Castagnola E, Cesaro S, Giacchino M, et al. Fungal infections in children with cancer: A prospective, multicenter surveillance study. Pediatr Infect Dis J 2006; 25: 634-639.

13. Montagna MT, Caggiano G, Lovero G, et al. Epidemiology of invasive fungal infections in the intensive care unit: Results of a multicenter Italian survey (AURORA Project). Infection 2013; 41: 645653.

14. Krcmery V, Laho L, Huttova M, et al. Aetiology, antifungal susceptibility, risk factors and outcome in 201 fungaemic children: Data from a 12-year prospective national study from Slovakia. J Med Microbiol 2002 51: 110-116.

15. Rosen GP, Nielsen K, Glenn S, Abelson J, Deville $\mathrm{J}$, Moore TB. Invasive fungal infections in pediatric oncology patients: 11-year experience at a single institution. J Pediatr Hematol Oncol 2005; 27: 135-140.

16. Wald A, Leisenring W, van Burik JA, Bowden RA. Epidemiology of aspergillus infections in a large cohort of patients undergoing bone marrow transplantation. J Infect Dis 1997; 175: 1459-1466.

17. Viscoli C, Girmenia C, Marinus A, et al. Candidemia in cancer patients: A prospective, multicenter surveillance study by the Invasive Fungal Infection Group (IFIG) of the European Organization for Research and Treatment of Cancer (EORTC). Clin Infect Dis 1999; 28: 10711079. 
18. Denning DW. Invasive aspergillosis. Clin Infect Dis 1998; 26: 781-803.

19. Pagano L, Girmenia C, Mele L, et al; GIMEMA Infection Program; Gruppo Italiano Malattie Ematologiche dell'Adulto. Infections caused by filamentous fungi in patients with hematologic malignancies. A report of 391 cases by GIMEMA Infection Program. Haematologica 2001; 86: 862-870.

20. Pongas GN, Lewis RE, Samonis G, Kontoyiannis DP. Voriconazole-associated zygomycosis: A significant consequence of evolving antifungal prophylaxis and immunosuppression practices? Clin Microbiol Infect 2009; 15(Suppl 5): 93-97.
21. Groll AH, Castagnola E, Cesaro S, et al; Fourth European Conference on Infections in Leukaemia; Infectious Diseases Working Party of the European Group for Blood Marrow Transplantation (EBMTIDWP); Infectious Diseases Group of the European Organisation for Research and Treatment of Cancer (EORTC-IDG); International Immunocompromised Host Society (ICHS); European Leukaemia Net (ELN). Fourth European Conference on Infections in Leukaemia (ECIL-4): Guidelines for diagnosis, prevention, and treatment of invasive fungal diseases in paediatric patients with cancer or allogeneic haemopoietic stem-cell transplantation. Lancet Oncol 2014; 15: e327-e340.

22. Hope WW, Castagnola E, Groll AH, et al; ESCMID Fungal Infection Study Group. ESCMID* guideline for the diagnosis and management of candida diseases 2012: Prevention and management of invasive infections in neonates and children caused by Candida spp. Clin Microbiol Infect 2012; 18(Suppl 7): 38-52. 